\title{
CTDI versus New AAPM Metrics to assess Doses in CT: a case study
}

\author{
M. C. S. Campelo ${ }^{\text {a; }}$ M. C. Silva ${ }^{\text {b; R. A. Terini }}{ }^{\text {c }}$ \\ ${ }^{a}$ Curso de Física, Pontifícia Universidade Católica de São Paulo, 01303-050, S. Paulo, SP, Brasil. \\ maria.macalidi@gmail.com \\ ${ }^{b}$ Setor de Física Médica, Hospital Israelita Albert Einstein (HIAE), 01308-050, São Paulo, SP, Brasil.. \\ marciac.silvag@gmail.com \\ ${ }^{c}$ Laboratório de Ensaios Não-Destrutivos (LABEND), Instituto de Energia e Ambiente, 05508-010, Universidade de \\ São Paulo, Cidade Universitária, S. Paulo, SP, Brasil. \\ ricardoaterini@gmail.com
}

\begin{abstract}
In modern CT, $\mathrm{CTDI}_{100}$ measurements would underestimate accumulated dose at the gantry center. AAPM TG 111 report proposed improved metrics for CT dosimetry, mainly for helical and wide beam width scanning. In this study, a methodology to assess CT dose, inspired on TG 111, was applied. Dosimeters were firstly calibrated in lab in beams like those utilized clinically. Using a reference $0.6 \mathrm{cc}$ Farmer chamber, two CT "pencil" chambers were calibrated in $\mathrm{P}_{\mathrm{KL}}$ by substitution method. Results showed differences $\leq 2 \%$ in the calibration coefficients, for three collimation apertures. A small $0.6 \mathrm{cc}$ chamber was calibrated in air kerma with this setup, without any collimator. After this, in a private Brazilian hospital, the small chamber was applied in dosimetry tests of a CT scanner, according to TG 111, determining Dose profiles and Equilibrium dose free-in-air $\left(\mathrm{D}_{\text {eq,air }}\right)$ for some protocols and pitch values. Results showed that $\mathrm{D}_{\text {eq,air }}$ increased when reducing pitch and Equilibrium dose-pitch product free in air (p. $\mathrm{D}_{\text {eq,air }}$ ) remain constant. In measurements with a $450 \mathrm{~mm}$ CT phantom, differences between Planar Average Equilibrium Dose $\left(\mathrm{D}_{\mathrm{eq}, \mathrm{p}}\right)$ and $\mathrm{CTDI}_{\mathrm{vol}}$ ranged between $30-37 \%$. This occurs because $\mathrm{CTDI}_{\mathrm{vol}}$ cannot include dose profile "tail" contribution, caused by scattering in phantom, especially for wide beam widths.
\end{abstract}

Keywords:

Computed tomography, AAPM TG 111, CTDI. 


\section{INTRODUCTION}

Computed tomography (CT) is among the largest sources of collective radiation dose. Absorbed dose in CT exams can be more than ten times higher than in other common radiographic procedures ${ }^{(1)}$. Smith-Bindman et al. $(2009)^{(2)}$ demonstrated that, for example, the median effective dose delivered through a single routine chest CT scan was equivalent to 119 ordinary chest radiograph series.

In $2008^{(3)}$, from more than 2 billion radiological exams (medical, dental, etc.) performed annually, 5\% were CT scans. This fraction, however, accounted for $34 \%$ of the world annual collective dose. Radiation exposure in CT has increased substantially over the past two decades due to the growing number of new installed scanners and CT procedures requests. It's a consensus that efforts need to be undertaken, including careful dosimetry, to minimize radiation risks and harms from CT, avoiding unnecessary studies, reducing the dose per study and the variation in dose across patients and facilities ${ }^{(2)}$.

CT dosimetry is generally performed using an ionization chamber with $100 \mathrm{~mm}$ length (the "pencil" chamber). First of all, to obtain reliable values, the utilized dosimeters should be properly calibrated in beams and setups like those measured clinically ${ }^{(1,4)}$. For this purpose, CT standard beams should be characterized in the dosimetry lab where the calibration should be performed.

To assess dose information in CT clinical procedures, pencil chambers are generally applied to obtain measurements both in air or inserted into cylindrical phantoms, to determine the CT Dose Index $\left(\mathrm{CTDI}_{100}\right)$. In the latter case, the chamber also detects scattered radiation generated in the phantom when irradiated, which is included in the total exposure of the patient. This requires that the response of the sensitive volume of the CT chamber be uniform throughout the axial length. Thus, first, the calibration methodology of such dosimeters should be revised in the light of the recent modalities and image acquisition technologies in $\mathrm{CT}^{(4)}$.

Furthermore, AAPM Task Group $111^{(5)}$ recently proposed improved metrics for CT dosimetry, using a small ion chamber, instead of the usual pencil one, positioned in a phantom long enough to establish dose equilibrium at the chamber location, mainly considering helical and 
multi-slice cone-beam scanning. Dosimetry in CT is commonly made obtaining $C T D I_{100}$ values, measured with a CT chamber inside a cylindrical phantom, in center and in periphery holes, in a single rotation and no table movement. This way, we can determine ${ }^{(1)}$ the weighted CTDI $\left(C T D I_{W}\right)$, the volumetric CTDI $\left(C T D I_{v o l}\right)$, and the air kerma-length product $\left(P_{K L}\right)$, related by:

$$
P_{K L}=C T D I_{\text {VOL }} \cdot L=\frac{C T D I_{W}}{\text { Pitch }} \cdot L
$$

Where $L$ is the scan length and the pitch factor is $b / n T$, with $b=$ table increment and $n T=$ total nominal width along the rotation axis.

Nevertheless, recent works have indicated that, in helical and multi-slice tomography with high number of rows, measured values of $\mathrm{CTDI}_{100}$ underestimate accumulated dose at center, as they do not include contributions of the dose profile "tail", caused by scattering in the phantom (or tissue). ${ }^{(6,7)}$

This study aimed to contribute to review the assessment of Dose in CT in Brazil and wherever needed, inspired on IAEA and AAPM recommendations ${ }^{(1,5)}$.

\section{MATERIALS AND METHODS}

\section{A. Improving the Calibration method: Setting a Farmer ion chamber as reference to cali- brate CT chambers}

Initially, CT standard beams (RQT) ${ }^{(1)}$ were characterized at the IEE-USP Non-Destructive Testing Laboratory (LABEND), using a calibrated $0.6 \mathrm{~cm}^{3}$ PTW Farmer chamber as reference. This chamber was used as the reference instead of a pencil chamber, as it is similar to the small CT chamber that would be applied in field measurements, according to AAPM recommendations. Then, we have calibrated two CT "pencil" chambers in $\mathrm{P}_{\mathrm{KL}}$ by the substitution method, with and without any collimator in front of the chamber to be calibrated ${ }^{(1,4)}$. In addition, we calibrated a Radcal CT $0.6 \mathrm{~cm}^{3}$ ion chamber in air kerma against the Farmer one.

Additionally, in other set of measurements, the homogeneity of a PTW pencil chamber response was checked as described below. 


\section{A.1. CT standard beams characterization}

Calibration measurements were made in the IEE/LABEND, using a constant potential Philips industrial X-ray equipment with an optical bench, a PTW monitor chamber and Pb collimators. The reference dosimeter was a $0.6 \mathrm{~cm}^{3}$ Farmer type PTW chamber (no buildup cap) and a PTW UNIDOS electrometer, both calibrated at the Radioprotection and Dosimetry Institute of the National Commission of Nuclear Energy (IRD/CNEN).

From previously characterized standard RQR beams with the reference Farmer chamber, CT specific beams (RQT 8, 9 and 10) were characterized, by adding suitable copper filtration ${ }^{(1,4)}$.

\section{A.2. Calibration of the CT chambers}

As a sequence, the calibration of two CT pencil type ion chambers (PTW and Radcal), connected to their electrometers, has been performed against the reference chamber in RQT beams, by the substitution method.

Each CT chamber were kept distant $0.97 \mathrm{~m}$ from the tube focal spot. Calibration was carried out in Air kerma-length product $\left(\mathrm{P}_{\mathrm{KL}}\right)$ in two ways: $(\mathrm{A})$ without any collimator, thus irradiating the whole chamber length, or (B) irradiating only its central portion, setting a reference $\mathrm{Pb}$ collimator $^{(1)}$ of aperture $2 \mathrm{~cm}$ (B.2) or $5 \mathrm{~cm}$ (B.5) which remained $0.90 \mathrm{~m}$ from the tube focus, in front of the chamber to be calibrated (Fig. 1). In each case, the aperture would represent, in lab, a scanning length used in a clinical CT procedure.

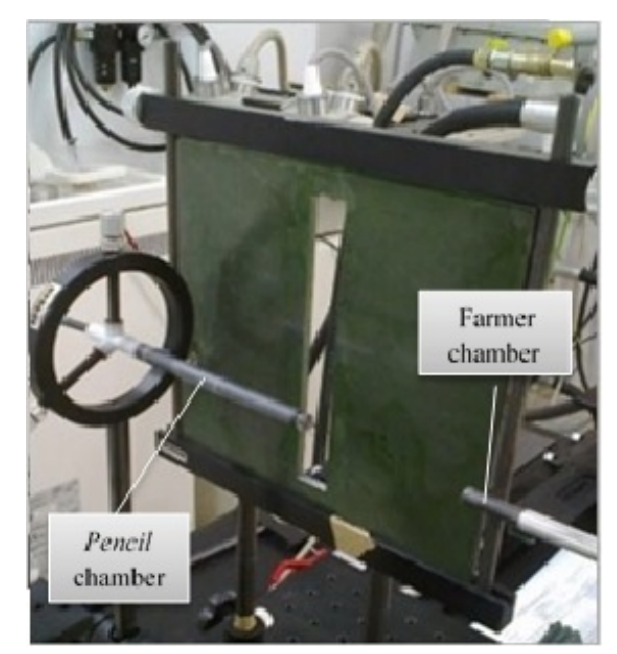

Figure 1 - Setup detail for the pencil CT chamber calibration against a Farmer reference chamber. 
In both methods, values of tube current, exposure time and Practical Peak Voltages $(\mathrm{PPV})^{(8,9)}$ have been set identical for each beam, and the readings have been corrected for standard air density and for relative position of the collimator. In each condition, $\mathrm{P}_{\mathrm{KL}}$ values and the calibration coefficients of each CT chamber were determined as

$$
N_{P K L-\text { user }}=\left(\frac{P_{K L-r e f}}{P_{K L-\text { user }}}\right)
$$

In the above expression, $P_{K L-r e f}$ and $P_{K L-u s e r}$ are the air kerma-length product determined, respectively, with the Farmer reference chamber, and with the chamber in calibration.

Finally, a 0.6cc ion chamber from Radcal Co. has been calibrated against the reference chamber in RQT beams, with no collimator, in terms of air kerma.

Additionally, the response homogeneity of one "pencil" chamber was checked. Thus, we shifted the chamber perpendicularly to the beam, behind the collimator, in steps of $1 \mathrm{~cm}$, and calibration procedure was repeated in each position with B.2 setup, for all RQT beams.

\section{B. Improving the Dosimetry of clinical CT scanners}

\section{B.1. Dose profiles}

In another step, the calibrated Radcal $0.6 \mathrm{cc}$ chamber was initially set aligned in air on $z$-axis of a Toshiba Aquilion One scanner, from a private Brazilian hospital, to measure dose profiles (Fig. 2). For this, an axial beam with nominal slice thickness $n T$ was used, in which measurements were taken with table increments of $1 \mathrm{~mm}$, in adjacent axial slices, so that the chamber covered a length $\mathrm{L}(\mathrm{mm})$, from $-\mathrm{L} / 2$ to $\mathrm{L} / 2$, large enough to include the scattering tail ${ }^{(7)}$. Three different $n T$ values were used: (a) $64 \times 0.5 \mathrm{~mm}$, (b) $80 \times 0.5 \mathrm{~mm}$ and (c) $4 \times 8 \mathrm{~mm}$. All measurements were made using $120 \mathrm{kV}, 400 \mathrm{~mA}$ and $0.5 \mathrm{sec}$ rotation time. 


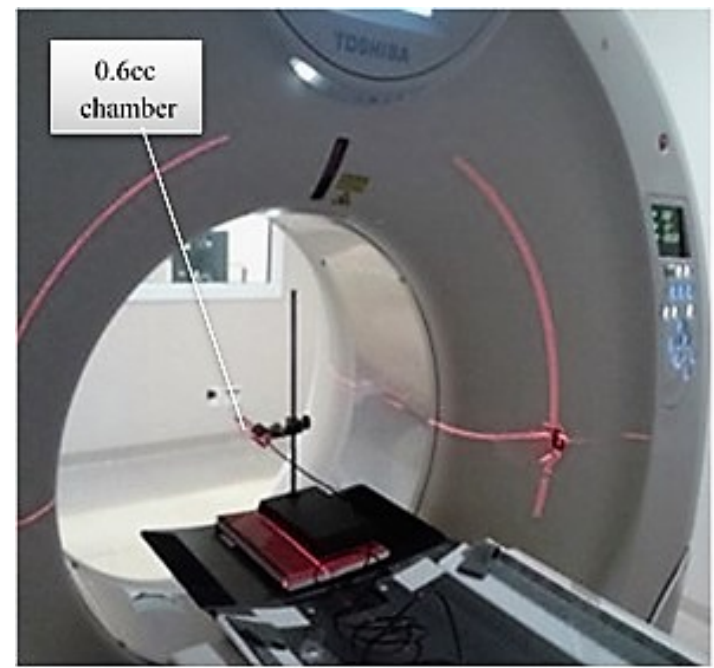

Figure 2 - Setup used to measure dose profiles, using a calibrated 0.6cc CT chamber, in zaxis of a Toshiba CT Scanner.

\section{B.2. Free-in-air Dose Measurements}

With the same setup and detector, values of Equilibrium dose-pitch product free-in-air (p.D eq,air $)$ and Equilibrium Dose free in air, $D_{\text {eq,ar }}{ }^{(5,10)}$, have been obtained, from the cumulative dose measured in air, but with continuous table movement, in helical acquisition from -L/2 to $\mathrm{L} / 2$, for various routine protocols, all with fixed $100 \mathrm{~mA}$ :

1) Skull: $\mathrm{nT}=32 \times 0.5=16 \mathrm{~mm}, 120 \mathrm{kV}, 0.75 \mathrm{~s}$ rotation, $75 \mathrm{mAs}$, FOV 220.2(S), Pitch: 1.406; $0.906 ; 0.844 ; 0.656, \mathrm{~L}=72 \mathrm{~mm}$;

2) Chest: $\mathrm{nT}=80 \mathrm{x} 0.5=40 \mathrm{~mm}, 120 \mathrm{kV}, 0.5 \mathrm{~s}$ rotation, 50mAs, FOV 400(L), Pitch: 1.388; $1.100 ; 0.813 ; 0.637, \mathrm{~L}=91 \mathrm{~mm}$;

3) Child abdomen: $\mathrm{nT}=64 \times 0.5=32 \mathrm{~mm}, 100 \mathrm{kV}, 0.5 \mathrm{~s}$ rotation, $50 \mathrm{mAs}$, FOV $320(\mathrm{M})$, Pitch: $1.484 ; 0.906 ; 0.828 ; 0.641, \mathrm{~L}=80 \mathrm{~mm}$.

The scan length L, for each protocol, was defined by Eq. (3) ${ }^{(5)}$ :

$$
L=l+n T+15 m m
$$

Where $l$ : Charge-collection length $(\mathrm{mm})$ of the ionization chamber (in this case, $21 \mathrm{~mm}$ ).

\section{B.3. CT equilibrium doses measured in the PMMA phantom}


In the third step, using the Radcal 0.6cc chamber and a large CT phantom (Fig. 3), values of Central Cumulative Dose, $\mathrm{D}_{\mathrm{L}}(0)$, were measured for $\mathrm{L}=50 \mathrm{~mm}$ to $450 \mathrm{~mm}-n T$, for two different abdomen protocols:

(a) $\mathrm{nT}=64 \times 0.5=32$; pitch 0.828 ; large focal spot; large bow tie filter; $400 \mathrm{~mA} ; 0.5 \mathrm{~s}$ rotation time; $120 \mathrm{kV}$.

(b) The same, but with $\mathrm{nT}=80 \mathrm{x} 0.5=40$ and pitch 0.813 .

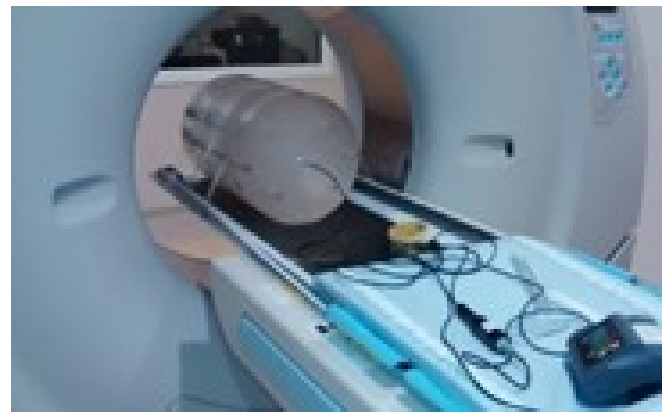

Figure 3 - Setup for the CT clinical measurements using the 0.6cc chamber (central position) and a $450 \mathrm{~mm}$ phantom (3 joined CT phantoms, $150 \mathrm{~mm}$ each).

With these values, a curve of $D_{L}(0)$ vs. $L$ was plotted and a best fit to the measured data was obtained applying Eq. $4^{(5)}$ :

$$
D_{L}(z=0)=h(L) \cdot D_{\text {eqq }}, \quad \text { where } h(L)=1-\propto, e^{-\left(\frac{4 L}{L_{e q}}\right)}
$$

Where $\mathrm{h}(\mathrm{L})$ is the approach to equilibrium function and $\alpha$ parameter relates to the scatter-toprimary ratio (SPR) along phantom axis.

This was performed in order to evaluate the Equilibrium cumulative dose $\left(D_{e q}\right)$, i.e., the saturation value of $\mathrm{D}_{\mathrm{L}}(\mathrm{z}=0)$ curve, and the Equilibrium scanning length $\left(\mathrm{L}_{\mathrm{eq}}\right)$, that is, the minimum scanning length L needed to obtain $D_{\text {eq. }}$. Equilibrium dose was determined for the central (c) and peripheral axes ( $\mathrm{Ph}$, with chamber in 12 o'clock position).

Finally, values of the Planar average equilibrium dose $\left(\mathrm{D}_{\mathrm{eq}, \mathrm{p}}\right)$ were calculated (assuming $\mathrm{D}_{\mathrm{eq}}$ proportional to $\mathrm{r}^{2}$ ) from Eq.(5), to be compared to the $\mathrm{CTDI}_{\mathrm{vol}}$ values informed by the CT scanner during examinations ${ }^{(5,10)}$.

$$
D_{e q ; p}=(1 / 2) \cdot D_{e q, \text { eentral }}+(1 / 2) \cdot D_{\text {eqperipheral }}
$$




\section{RESULTS AND DISCUSSIONS}

\section{A. Calibration of CT chambers against a Farmer one in CT standard beams}

\section{A.1. CT standard beams characterization}

RQT beams have been characterized for PPV values ${ }^{(8,9)}$ invasively determined with deviations $\leq 0.10 \mathrm{kV}$, and for $1^{\text {st }}$. HVL values obtained with differences $\leq 0.04 \mathrm{mmAl}$ from standard reference values $^{(1)}$, as appearing in $2^{\text {nd }}$ and $3^{\text {rd }}$ columns of Table 1 below.

\section{A.2. CT chamber calibrations}

Table 1 summarizes, for example, the results obtained for the calibration coefficients of the 100mm PTW CT chamber, together with beam characterization parameters. Results for the Radcal pencil chamber were similar. Results showed differences up to $2 \%$ in the obtained coefficients, for all the analyzed HVL values (6.94 to $10.13 \mathrm{mmAl}$ ), depending on the collimator aperture. When using collimator, the obtained coefficients are systematically lower, but differences remain inside uncertainties range. Finally, last right column shows the calibration data, in air kerma, obtained for the $0.6 \mathrm{cc}$ Radcal chamber, for the same standard beams, with no collimator.

Table 1 - Parameters obtained in a 100mm CT chamber calibration in $\mathrm{P}_{\mathrm{KL}}$ against the Farmer reference chamber, for RQT beams ${ }^{(1)}$, with and without collimation (methods B and A). The last right column shows calibration data, in air kerma, obtained for the $0.6 \mathrm{cc}$ Radcal chamber for the same beams, with no collimator.

\begin{tabular}{ccccccc}
\hline $\begin{array}{c}\text { Standard } \\
\text { Beam }\end{array}$ & $\begin{array}{c}\text { PPV } \\
(\mathrm{kV})\end{array}$ & $\begin{array}{c}\text { HVL } \\
(\mathrm{mmAl})\end{array}$ & $\begin{array}{c}\text { NPKL-user } \\
(\mathbf{A})\end{array}$ & $\begin{array}{c}\text { NPKL-user-100 } \\
(\mathbf{B . 2})(\mathrm{L}=2 \mathrm{~cm})\end{array}$ & $\begin{array}{c}\text { NPKL-user-100 } \\
(\mathbf{B . 5})(\mathrm{L}=5 \mathrm{~cm})\end{array}$ & $\begin{array}{c}\mathbf{N}_{\text {k-user-0.6 }} \\
\text { (no collimator) }\end{array}$ \\
\hline RQT 8 & 100.07 & 6.90 & $1.003(27)$ & $0.983(20)$ & $0.982(17)$ & $0.974(34)$ \\
RQT 9 & 120.03 & 8.40 & $1.023(26)$ & $1.001(19)$ & $1.004(16)$ & $0.985(34)$ \\
RQT 10 & 149.80 & 10.10 & $1.008(27)$ & $0.987(19)$ & $0.992(17)$ & $0.986(34)$ \\
\hline
\end{tabular}

In the homogeneity tests, results show that the response of both "pencil" chambers changed slowly $(\sim 3 \%$ in $3.0 \mathrm{~cm})$, along the lateral positions behind the $2 \mathrm{~cm}$ aperture collimator, for all RQT standard beam, but homogeneity was just verified until $\sim 3.5 \mathrm{~cm}$ from chamber center, considering uncertainties. 


\section{B. Dosimetry of a clinical CT scanner}

\section{B.1. Determination of Dose profiles (in air):}

Fig.4 shows dose profiles obtained for the axial protocol applied with the Toshiba scanner, for different nominal slice thicknesses nT. Experimental beam widths have been obtained as the profile FWHM, determined as accurately as possible.

The obtained values were: (a) $37.8(25) \mathrm{mm}, 18 \%$ higher than the nominal width $\mathrm{nT}$; (b) 47.2(25) mm, 18\% higher than nT and (c) 34.3(25) mm, 7\% higher than $\mathrm{nT}$.
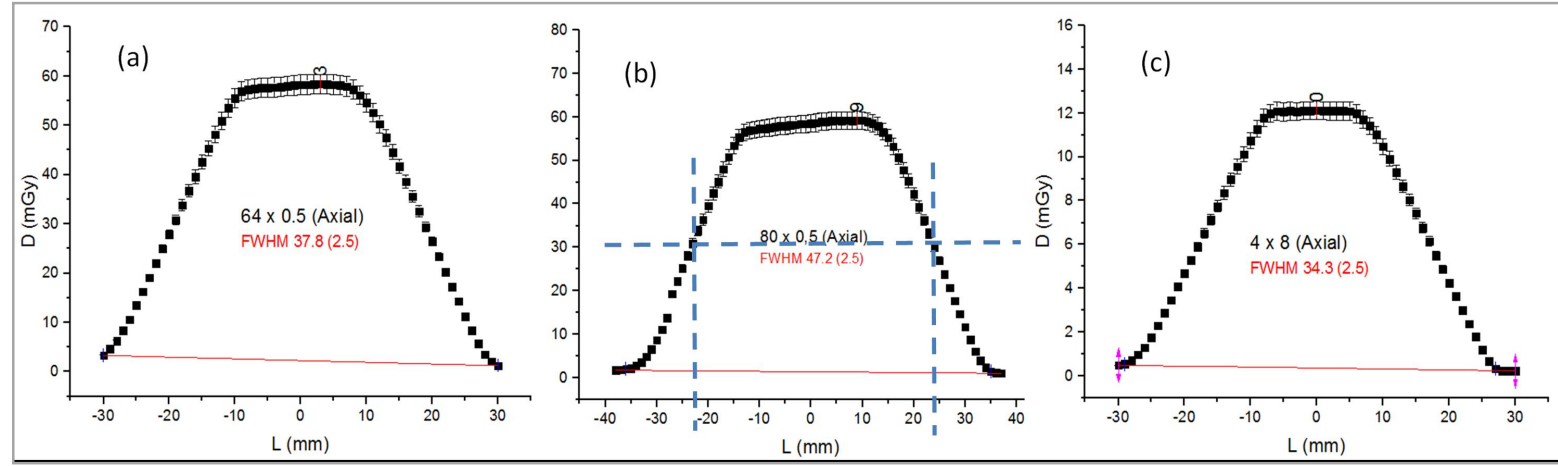

Figure 4 - Dose profile measured with the CT 0.6cc Radcal chamber, for axial protocols in a Toshiba Scanner, with $n T=$ (a) $64 \times 0.5=32 \mathrm{~mm}$; (b) $80 \times 0.5=40 \mathrm{~mm}$ and (c) $4 \times 8=32 \mathrm{~mm}$. Blue traces are to determine FWHM.

Therefore, in this survey of dose profiles, slice thicknesses were 7 to $18 \%$ higher than the nominal values, due to scattering in the phantom.

\section{B.2. Evaluation of Equilibrium Doses free in air}

From the cumulative dose measured in air, values of $p . D_{\text {eq,air }}$ and $D_{\text {eq,air }}$ were determined ${ }^{(5)}$. Table 2 shows that $D_{\text {eq,air }}$ values increase as the pitch is reduced, while $p . D_{\text {eq,air }}$ values remain constant. 
Table 2 - Values of $\mathrm{D}_{\text {eq,ar }}$ and $p \mathrm{D}_{\text {eq,ar }}$, normalized by $\mathrm{mAs}$, obtained in three analyzed protocols, for four pitch modes of equipment operation: detail (dt), standard (std), other (ot) and fast $(\mathrm{ft})$.

\begin{tabular}{|c|c|c|c|c|c|c|c|c|}
\hline \multicolumn{3}{|c|}{ Skull } & \multicolumn{3}{|c|}{ Chest } & \multicolumn{3}{|c|}{ Child Abdomen } \\
\hline Pitch & $\begin{array}{c}\mathbf{D}_{\text {eq,ar }} \\
(\mathrm{mGy} / \mathrm{mAs})\end{array}$ & $\begin{array}{c}\mathbf{p D}_{\text {eq,ar }} \\
(\mathrm{mGy} / \mathrm{mAs})\end{array}$ & Pitch & $\begin{array}{c}\mathbf{D}_{\text {eq,ar }} \\
(\mathrm{mGy} / \mathrm{mAs})\end{array}$ & $\begin{array}{c}\mathbf{p D}_{\text {eq,ar }} \\
(\mathrm{mGy} / \mathrm{mAs})\end{array}$ & Pitch & $\begin{array}{c}\mathbf{D}_{\text {eq,ar }} \\
(\mathrm{mGy} / \mathrm{mAs})\end{array}$ & $\begin{array}{c}\mathbf{p D}_{\text {eq,ar }} \\
(\mathrm{mGy} / \mathrm{mAs})\end{array}$ \\
\hline $\begin{array}{c}\mathrm{dt} \\
0.656\end{array}$ & $151.82(8)$ & $98.13(6)$ & $\begin{array}{c}\mathrm{dt} \\
0.637\end{array}$ & $89.95(6)$ & $56.46(4)$ & $\begin{array}{c}\mathrm{dt} \\
0.641\end{array}$ & $74.03(11)$ & $46.22(7)$ \\
\hline $\begin{array}{c}\text { std } \\
0.844\end{array}$ & $118.11(5)$ & $98.21(5)$ & $\begin{array}{c}\text { std } \\
0.813\end{array}$ & $75.75(6)$ & $56.68(5)$ & $\begin{array}{c}\text { std } \\
0.828\end{array}$ & $57.31(5)$ & $46.22(11)$ \\
\hline $\begin{array}{c}\text { ot } \\
0.906\end{array}$ & $110.13(5)$ & $98.31(5)$ & $\begin{array}{c}\text { ot } \\
1.100\end{array}$ & $52.55(4)$ & $56.96(4)$ & $\begin{array}{c}\text { ot } \\
0.906\end{array}$ & $52.37(5)$ & $46.21(5)$ \\
\hline $\begin{array}{c}\mathrm{ft} \\
1.406\end{array}$ & $70.96(7)$ & $98.30(9)$ & $\begin{array}{c}\mathrm{ft} \\
1.388\end{array}$ & 41.67(18) & $56.99(24)$ & $\begin{array}{c}\mathrm{ft} \\
1.484\end{array}$ & $32.10(5)$ & $46.40(7)$ \\
\hline
\end{tabular}

\section{B.3. Assessment of the dose delivered in CT procedures (in phantom).}

Fig. 5 shows an example of $D_{L}(0)$ vs. L graph, with a best fit of the approximation function to equilibrium $h(\mathrm{~L})$ (Eq. 4) to the measured central and peripheral values of $\mathrm{D}_{\mathrm{L}}(0)$. From these curves, values of $D_{e q}$ and $L_{e q}$ have been determined for each chamber position in the phantom and analyzed protocol. 


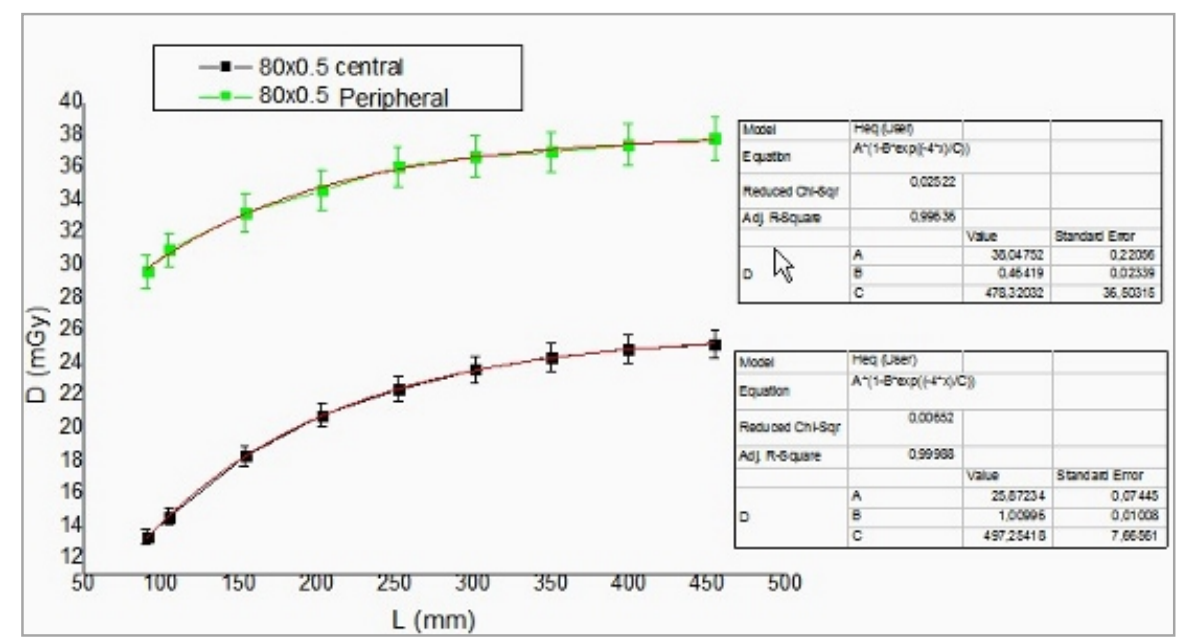

Figure 5 - Approach to equilibrium of Cumulative Dose $D_{L}(0)$ in central and peripheral axis, for a beam with 40mm (80x0.5) thickness, in (b) helical protocol (see B.3). Red lines are the approximation function to equilibrium.

Curves of approach to equilibrium have been obtained for the $80 \times 0.5$ and for the $64 \times 0.5$ protocols. Table 3 shows collected data at central (c) axis in the phantom, and at peripheral axis, discriminating mean (me), maximum $(\mathrm{mx})$ and minimum $(\mathrm{mn})$ obtained values. These variations occur because, in periphery measurements, tube rotation can start in different positions, influencing the collected data.

From $D_{e q}$ (central and peripheral medium, maximum and minimum), values of Planar Average Equilibrium Dose $\left(D_{\text {eq,p }}\right)$ were calculated ${ }^{(5)}$. Table 3 shows that, as expected, $\mathrm{D}_{\text {eq,p }}$ exceeds $\mathrm{CTDI}_{\mathrm{vol}}$, differences ranging between $30-37 \%$.

$\mathrm{CTDI}_{\mathrm{vol}}$ values have been obtained from the panel of the CT equipment, which has been checked preliminarily by routine quality control tests, presenting accuracy $\leq 5 \%$. 
Table 3 - Approach to equilibrium function data collected from the curves, for both measured protocols, $80 \times 0.5$ and $64 \times 0.5$, including the values for $\mathrm{D}_{\mathrm{L}}, \alpha, \mathrm{L}_{\mathrm{eq}}$ and $\mathrm{R}^{2}$. Columns at right show the calculated values of Planar Average Equilibrium Dose, $\mathrm{D}_{\mathrm{eq}, \mathrm{p}}$ (Eq.5), and their difference $(\Delta \%)$ to the CTDIvol given by equipment.

\begin{tabular}{|c|c|c|c|c|c|c|c|c|}
\hline Protocols & $\underset{(\mathrm{mGy})}{\mathbf{D}_{\mathbf{L}}}$ & $\alpha$ & $\begin{array}{c}\mathbf{L}_{\mathbf{e q}} \\
(\mathrm{mm})\end{array}$ & $\mathbf{R}^{2}$ & $\begin{array}{l}\text { Parameters to } \\
\text { Calculate } D_{\text {eq,p }}\end{array}$ & $\begin{array}{l}\mathbf{D}_{\text {eq,p }} \\
(\mathrm{mGy})\end{array}$ & $\begin{array}{l}\text { CTDIvol } \\
\text { (Equipment) } \\
\text { (mGy) }\end{array}$ & $\begin{array}{c}\Delta \\
(\%)\end{array}$ \\
\hline $80 \times 0.5(c)$ & $25.87(7)$ & $1.01(1)$ & $497(80)$ & 1.00 & - & & & \\
\hline $80 \times 0.5$ (me) & $36.72(30)$ & $0.64(8)$ & $354(45)$ & 0.99 & $80 \times 0.5$ (c-me) & $31.30(27)$ & 23.4 & $34 \%$ \\
\hline $80 \times 0.5(m x)$ & $38.05(22)$ & $0.46(2)$ & $478(37)$ & 1.00 & $80 x 0.5(c-m x)$ & $31.96(21)$ & 23.4 & $37 \%$ \\
\hline $80 \times 0.5(\mathrm{mn})$ & $35.69(42)$ & $0.75(16)$ & $318(59)$ & 0.97 & $80 \times 0.5(c-m n)$ & $30.78(37)$ & 23.4 & $32 \%$ \\
\hline $64 \times 0.5(c)$ & $28.27(7)$ & $1.00(1)$ & $497(50)$ & 1.00 & - & & & \\
\hline $64 \times 0.5$ (me) & $39.59(44)$ & $0.48(5)$ & $378(60)$ & 0.98 & $64 \times 0.5$ (c-me) & $33.93(38)$ & 25.5 & $33 \%$ \\
\hline $64 \times 0.5(\mathrm{mx})$ & $41.22(44)$ & $0.41(2)$ & $506(63)$ & 0.99 & $64 \times 0.5(c-m x)$ & $34.74(38)$ & 25.5 & $36 \%$ \\
\hline $64 \times 0.5(\mathrm{mn})$ & $37.85(56)$ & $0.99(35)$ & $211(55)$ & 0.95 & $64 \times 0.5(c-m n)$ & $33.06(50)$ & 25.5 & $30 \%$ \\
\hline
\end{tabular}

\section{CONCLUSIONS}

It is possible to conclude, first, that the collimation of the CT pencil chamber under test has some influence on the calibration result, which is in agreement with the recommendations of IAEA $^{(1)}$ and Hourdakis et al. ${ }^{(11)}$, based on measurements made in "good geometry".

The growing number of CT exams in Brazil requires, indeed, the calibration of the CT chambers utilized for dosimetry and quality control tests in reference beams such as those utilized in the clinical practice. This work reinforces this necessity and indicates the possibility of use small chambers as references to other small ones as well as to CT "pencil" chambers.

The use of a Farmer-like chamber in CT for dose measurements is a more suitable alternative for helical scans and wide beam width scanners (like multislice units with many rows), allowing, 
for example, the survey of dose profiles. In the present work, slice thicknesses were evaluated from the profiles and their values were 7 to $18 \%$ higher than the nominal values.

Moreover, $\mathrm{CTDI}_{\mathrm{vol}}$ values, as informed by the CT equipment, were lower than Planar average equilibrium doses $\left(D_{\text {eq,p }}\right)$ values, determined in agreement with AAPM TG111 $1^{(5)}$ report, differences ranging between $30-37 \%$ (for $\mathrm{nT}=40 \mathrm{~mm}$ and $32 \mathrm{~mm}$ ). Results were similar in acceptance tests made with another Toshiba CT scanner in the same hospital. These results are also in agreement with the findings of Descamps et al. ${ }^{(10)}$, obtained using a Siemens CT scanner, and of Dixon and Ballard ${ }^{(7)}$, who used two GE scanners.

Differences between values of $\mathrm{CTDI}_{\mathrm{vol}}$ and $\mathrm{D}_{\mathrm{eq}, \mathrm{p}}$ are also consistent with results of Monte Carlo simulations made by Boone ${ }^{(12)}$ showing that $\mathrm{CTDI}_{\mathrm{vol}}$ systematically underestimates dose and thus seems to be no longer suitable for determining the absorbed dose during a CT examination, as stands AAPM TG111 report ${ }^{(5)}$.

Other groups have been trying to investigate ways of still avail the dose index values reported by the scanners to evaluate the new dose quantities ${ }^{(13)}$. Besides, teams like Platten et al. ${ }^{(14)}$, from IPEM (UK), are appraising some alternatives to replace the usual $\mathrm{CTDI}_{\mathrm{vol}}$, as reported (with no AEC), by AAPM methodology or even by a simpler upgrade in the original method.

As originally proposed, $\mathrm{CTDI}_{\mathrm{vol}}$ is still a useful index to track across patients, protocols and scanners for quality assurance purposes, but indeed, it is clearly not the value of patient dose $\mathrm{e}^{(5,10)}$.

An additional warning is that not all radiologists are realizing this fact, just because the lack of adequate education in radiation physics. As comments Dr. Carvalho, A.C.P. ${ }^{(15)}$, based on large teaching experience, "sometimes unnecessary imaging studies are frequently requested because the assisting physician does not have in mind that the radiation dose delivered by a chest CT scan corresponds to the dose delivered by approximately 200 to 500 chest radiographies, not to mention the much higher cost of computed tomography."

After all, we tend to agree with him, when he conclude that, "it is necessary to rethink the curricular reforms, the modern pedagogy, distance teaching and learning, the internet, the tablets, and seriously thinking of resuming the education of physicians enabling them to care for human beings."(15) 


\section{AKNOWLEDGEMENT}

We wish to acknowledge to LABEND of IEE-USP and to Hospital Israelita Albert Einstein de São Paulo by the use of their facilities, and also to the Brazilian agencies FAPESP and CNPq by the partial financial support.

\section{REFERENCES}

1 IAEA - International Atomic Energy Agency. Dosimetry in Radiology: An International Code of Practice. Technical Reports Series $\mathbf{n}^{\circ}$ 457. Vienna: IAEA, 2007.

2 SMITH-BINDMAN R. et al.. Radiation dose associated with common computed tomography examinations and the associated lifetime attributable risk of cancer. Arch. Intern. Med., v.169(22), p. 2078-2086, 2009.

3 UNSCEAR - United Nations Scientific Committee on the Effects of Atomic Radiation. Sources and Effects of Ionizing Radiation. UNSCEAR 2008 Report, vol. I, Annex A: Medical Radiation Exposures, NY: UNSCEAR, 2010.

4 MERIMAA HK, TAPIOVAARA M, KOSUNEN A, TOROI P. Calibration and features of air kerma-length product meters. Rad. Prot. Dos., v. 152(4), p. 384-392, 2012.

${ }^{5}$ AAPM - American Association of Physics in Medicine. Comprehensive methodology for the evaluation of radiation dose in X-ray computed tomography. AAPM report $\mathrm{n}^{\circ}$. 111, Task Group 111. College Park, MD: AAPM, 2010.

${ }^{6}$ BOONE J M. The trouble with CTDI100. Med. Phys., v. 34, p. 1364-1371, 2007.

${ }^{7}$ DIXON R L. and BALLARD, A. C.. Experimental validation of a versatile system of CT dosimetry using a conventional ion chamber: Beyond CTDI100. Med. Phys., v. 34, p. 3399$3413,2007$.

${ }^{8}$ KRAMER HM, SELBACH HJ, ILES WJ. The Practical Peak Voltage of Diagnostic X-Ray Generators, Br. J. Radiol., v. 71, p. 200-209, 1998. 
9 TERINI RA, POTIENS, MPA, HERDADE, SB, PEREIRA, MAG, PIRES, JSJ, VIDEIRA, HS. Measurement of the quantity Practical Peak Voltage in the radiology practice. Radiologia Brasileira, v. 42, p. 389-394, 2009.

${ }^{10}$ DESCAMPS C, GONZALEZ M. Measurements of the dose delivered during CT exams using AAPM Task Group Report No. 111, Journal of Applied Clinical Medical Physics, vol. 13, N. 6, 2012.

${ }^{11}$ HOURDAKIS CJ, BOZIARI A, KOUMBOULI E, Calibration of pencil type ionization chambers at various irradiation lengths and beam qualities. Proceedings IAEA IDOS 2010, vol.2, 21-33, IAEA STI/PUB/1514, 2010.

12 BOONE JM, Dose spread functions in computed tomography: A Monte Carlo study. Med. Phys., v. 36, p. 4547-4554, 2009.

${ }^{13}$ LI X, ZHANG D, LIU B, Calculations of two new dose metrics proposed by AAPM Task Group 111 using the measurements with standard CT dosimetry phantoms. Med. Phys., v. 40, 081914, 2013. Available at http://dx.doi.org/10.1118/1.4813899.

${ }^{14}$ PLATTEN DJ et al. Radiation dosimetry for wide-beam CT scanners: recommendations of a working party of the Institute of Physics and Engineering in Medicine, Br. J. Radiol., 86:20130089, 2013.

15 CARVALHO ACP. Medical knowledge of ionizing radiations, Radiol Bras. v. Mar/Abr. 47(2):X. 2014. Available at http://dx.doi.org/10.1590/S0100-39842014000200004 . 